\title{
Usefulness of mean platelet volume to platelet count ratio for predicting the risk of mortality in community- acquired pneumonia
}

Jooyoung Cho, Saejin Lee, Young Uh, Jong-Han Lee

Department of Laboratory Medicine, Yonsei University Wonju College of Medicine, Wonju, South Korea

Submitted: 23 July 2019

Accepted: 27 October 2019

Arch Med Sci 2020; 16 (6): 1327-1335

DOI: https://doi.org/10.5114/aoms.2020.92404

Copyright $\odot 2020$ Termedia \& Banach

\section{Abstract}

Introduction: The association between mean platelet volume (MPV) to platelet count $(P C)$ ratio and prognosis has been demonstrated in some diseases but not in community-acquired pneumonia (CAP). In this study, we evaluated the ability of MPV to PC ratio (MPR) to predict short-term mortality in CAP patients.

Material and methods: We retrospectively analysed data archived over 10 years and stratified MPR values into quartiles. Relations between MPR (femtoliters/number of thousand platelets per microlitre) quartiles and 60-day mortality were examined. Logistic regression was performed to adjust for confounders, and the Kaplan-Meier method was used for survival analysis. Results: After adjusting for confounding factors, the odds ratios of 60-day mortality for CAP were 2.66 ( $95 \% \mathrm{Cl}: 2.04-3.46)$ for the fourth MPR quartile (range $\geq 5.19 ; p<0.001$ ) versus the first MPR quartile (range $\leq 2.45$ ). KaplanMeier curves indicated that a higher MPR was associated with a higher risk of mortality among CAP patients, and this was confirmed by the log-rank test $(p<0.001)$.

Conclusions: Mean platelet volume to PC ratio was found to be positively correlated with short-term mortality. Our data indicate that MPR might be a significant predictive marker of the mortality in CAP. Further prospective studies are required to establish the exact role of MPR in CAP and other diseases.

Key words: mean platelet volume to platelet ratio, mortality, communityacquired pneumonia.

\section{Introduction}

Community-acquired pneumonia (CAP) is a leading cause of death worldwide [1-4]. Its annual incidence is $0.3-0.6 \%$ in adults [4, 5], and it accounts for $5-15 \%$ of deaths among hospitalised patients and nearly 2 million deaths annually $[5,6]$. Accordingly, early risk assessment and prognostic prediction are necessary to reduce healthcare-related costs and disease-related mortality [7-9]. Many studies have assessed means of predicting disease severity and outcomes to enable adequate allocation of healthcare resources and optimal treatment. Several efforts have been made to identify potential predictors of prognosis in CAP $[3,5,6$, 9-15]. Currently, pneumonia severity scales such as CURB-65 (confusion, urea, respiration, blood pressure, and age $>65$ years) and the pneumo-

\author{
Corresponding author: \\ Jong-Han Lee MD, PhD \\ Department \\ of Laboratory Medicine \\ Wonju Severance Christian \\ Hospital \\ Yonsei University Wonju \\ College of Medicine \\ 20 llsan-ro \\ Wonju 26426, South Korea \\ Phone +82 337411594 \\ Fax: +82 337310506 \\ E-mail: cello425@yonsei.ac.kr
}


nia severity index (PSI) are widely used to predict prognosis. In addition, the prognostic values of procalcitonin and C-reactive protein (CRP), both biomarkers of infectious diseases, have also been studied in this respect $[1,2,10-14,16,17]$. Others have compared the utility of white blood cell (WBC) counts versus procalcitonin or CRP $[18,19]$ or studied the prognostic values of neutrophil and lymphocyte counts in CAP [8, 20, 21].

As well as these haematological parameters, platelet indices have been studied with respect to the prediction of prognosis, disease severity, and mortality in many diseases. In one study, thrombocytopaenia and thrombocytosis were found to be strongly associated with short-term mortality in CAP patients [22]. In another multicentre observational study, it was demonstrated that thrombocytopaenia independently predicted mortality in CAP patients admitted to an intensive care unit (ICU) [23]. In a recent study, a relationship was observed between mean platelet volume (MPV) and short-term mortality in severe sepsis and/or septic shock patients [24]. Also, one study suggested that MPV might be a useful predictor for CAP diagnosis in paediatric patients [25].

Higher values of MPV and lower platelet counts (PCs) have been investigated in previous studies, and the MPV to PC ratio (MPR) has also been studied in many diseases. However, there has been no study on the relationship between MPR and mortality in CAP patients.

We undertook this study to determine the predictive value of MPR in CAP; (1) by evaluating the ability of MPR to predict short-term mortality, and (2) by comparing MPR values with pneumonia severity scales (CURB-65 and PSI).

\section{Material and methods}

\section{Study design and data collection}

We retrospectively reviewed the electronic medical records of all patients with a diagnosis of CAP admitted to Wonju Severance Christian Hospital (a tertiary university-affiliated hospital located in Wonju, South Korea) during the 10-year period from February 2008 to January 2018. Patients were diagnosed as CAP if they presented one of the following signs or symptoms; fever $>38.0^{\circ} \mathrm{C}$, cough, sputum, dyspnoea, pleuritic chest pain, or auscultatory findings of abnormal breath sounds plus new infiltrate by chest radiography within 48 h of hospitalisation $[9,17,26,27]$. Patients with a history of hospitalisation within the 2 weeks prior to current admission were excluded. During the study period, 3665 patients received a diagnosis of pneumonia, and 150 patients had sufficient data to allow us to calculate CURB-65 and PSI scores according to the Infectious Diseases Society of America/American Thoracic Society (IDSA/ATS) guidelines [28].

The following demographic and clinical data were extracted: age, gender, systolic/diastolic blood pressure, respiratory rate, pulse rate, body temperature, mental status, bacteraemia, ICU admission, date of admission and death, presence of risk factors (history of hypertension, diabetes mellitus, cerebrovascular accident, heart failure, coronary artery occlusive disease, hepatic disease, renal disease, and malignancy), and accompanying respiratory diseases (asthma, bronchitis, chronic obstructive pulmonary disease, and tuberculosis). The following laboratory data were collected: WBC count, haemoglobin, platelet count, mean corpuscular volume (MCV), MPV, delta neutrophil index (DNI), erythrocyte sedimentation rate (ESR), serum CRP, and procalcitonin.

For all patients enrolled in this study throughout the experiment, both MPV (reference range: 6.4-9.7 fl) and PC (reference range: $165-360 \times 10^{3} / \mu \mathrm{l}$ ) values were routinely measured using an ADVIA 2120i automated haematology analyser (Siemens Healthineers, Erlangen, Germany). The relationship between MPV to PC ratio (fl/number of thousand platelets per microlitre) and short-term (60-day) mortality was examined. All study subjects were followed up for more than 60 days.

This study was performed after obtaining approval from the institutional review board (IRB) of Wonju Severance Christian Hospital (IRB no. CR318070), which waived the requirement for informed consent.

\section{Statistical analysis}

For categorical data, data distributions are presented as frequencies and percentages and compared using the $\chi^{2}$ test. The Kolmogorov-Smirnov test was used to confirm normality for numerical data, and a $p$-value greater than 0.05 indicates normal distribution of data (parametric data). For parametric data, results are presented as means \pm standard deviations (SDs), and comparisons were performed using Student's $t$-test, and for non-parametric data, results are presented as medians and interquartile ranges (IQRs), and comparisons were made using the Mann-Whitney $U$ test.

Receiver operating characteristic (ROC) curve analysis was used to compare the abilities of various parameters, such as WBC, DNI, ESR, CRP, procalcitonin, neutrophil to lymphocyte ratio, and MPR, to predict 60-day mortality. Predictive values were determined by area under the curve (AUC) analysis. Patients were classified into MPR quartiles and compared. Results for numeric data were compared using analysis of variance (ANOVA) or the Kruskal-Wallis test (for parametric or nonparametric data, respectively), and when signifi- 
cant differences were found, Tukey's adjustment was used for pairwise comparisons. Univariate and multivariate logistic regression analyses were performed before and after adjusting for confounders. Survival analysis was performed using the Kaplan-Meier estimation, and statistical significance was confirmed using the log-rank test. In addition, the abilities of CURB-65, PSI, and MPR to predict disease mortality, bacteraemia rates and ICU admission rates were compared, and sensitivities, specificities, and AUC values were determined.

The analysis was performed using SPSS version 23.0 (IBM Corp., Armonk, NY, USA) and Analyse-it version 5.01 (Analyse-it Software, Ltd., Leeds, UK) in Microsoft Excel 2010 (Microsoft Corp, Redmond, Washington, USA). Statistical significance was accepted for $p$-values $<0.05$.

\section{Results}

\section{Baseline characteristics}

Baseline characteristics of the 3665 study subjects are provided in Table I. Among them 2351 (64.1\%) were men and 1314 (35.9\%) were women, and the overall median subject age was 74.0 years (interquartile range (IQR), 65.0-80.0). Short-term survival (60-days) was evaluated for all patients, and 591 (16.1\%) patients died within the 60-day follow-up period. In terms of medical histories, hypertension, asthma, and chronic obstructive pulmonary disease (COPD) were more frequent ( $p<0.001,0.002$, and 0.023, respectively) and both systolic and diastolic blood pressure were higher ( $p<0.001$ and 0.025 , respectively) in survivors. However, malignancy was more frequent ( $p=0.009$ ) in non-survivors. In addition, altered mental status rate, bacteraemia rate, and ICU admission rate were higher for non-survivors ( $p=0.024,<0.001$, and $<0.001$, respectively).

Regarding laboratory findings, DNI and procalcitonin levels were significantly higher in nonsurvivors ( $p<0.001$ for all). And we found that MPV was significantly higher $(p<0.001)$ and PC was significantly lower $(p<0.001)$ in non-survivors.

\section{Predictive ability of mean platelet volume to platelet count ratio}

ROC curve analysis showed MPR had an AUC for 60-day mortality of 0.63 (95\% confidence interval $(\mathrm{Cl}): 0.60-0.65)$, which was higher than that of any other variable except for the procalcitonin level (AUC of $0.65,95 \% \mathrm{Cl}$ : 0.63-0.67) examined in the present study. The AUCS of WBC, DNI, CRP, $\mathrm{ESR}$, and neutrophil to lymphocyte ratio were 0.54 (95\% Cl: 0.51-57), 0.61 (95\% Cl: 0.59-0.64), 0.52
(95\% Cl: 0.49-0.55), and 0.54 (95\% Cl: 0.52-0.57), respectively (Table $\mathrm{II}$ ).

\section{Patient characteristics according to mean platelet volume to platelet count ratio quartiles}

MPV to PC ratio was calculated as MPV (fl) divided by PC (number of thousand platelets/ $\mu \mathrm{l}$ ). We stratified the 3665 study subjects into MPR quartiles as follows: first quartile $0.00-2.45$, second quartile 2.46-3.50, third quartile 3.51-5.18, and fourth quartile $\geq 5.19$. A summary of patient characteristics by MPR quartile is provided in Table III. The ICU admission rate was significantly increased according to MPR quartile $(p<0.001)$, whereas altered mental status and bacteraemia rate showed no significant tendency to increase ( $p=0.217$ and 0.716 , respectively). Regarding laboratory findings, WBC count, PC, and ESR showed a significant tendency to decrease $(p<0.001$ for all), and MPV showed a significant tendency to increase $(p=0.044)$.

\section{Survival analysis according to mean platelet volume to platelet count ratio}

For all study subjects, 60-day all-cause mortality was $16.1 \%$ (591/3665). A summary of univariate and multivariate logistic regression results obtained before and after adjusting for possible confounders is provided in Table IV. Univariate logistic regression analysis for the prediction of 60-day mortality revealed that patients in the the third and fourth MPR quartiles had higher mortality rates than patients in the first quartile. Odds ratios (ORs) with respect to the first quartile were as follows: 1.16 (95\% Cl: 0.87-1.55) for the second quartile ( $p=0.307), 1.48$ (95\% Cl: $1.12-1.95)$ for the third ( $p=0.006)$, and 2.93 (95\% Cl: 2.27-3.78) for the fourth $(p<0.001)$. Multivariate logistic regression adjusted for age and medical history produced similar findings: OR of $1.49(95 \% \mathrm{Cl}$ : $1.13-1.97)$ for the third quartile $(p=0.005)$ and 3.06 (95\% Cl: $2.36-3.96)$ for the fourth $(p<0.001)$. After additional adjustment for laboratory findings, such as WBC, ESR, DNI, and procalcitonin, the ORs for 60-day mortality were $1.37(95 \% \mathrm{Cl}$ : $1.03-1.81)$ for the third quartile $(p=0.030)$ and 2.66 (95\% Cl: 2.04-3.46) for the fourth ( $p<0.001)$.

Kaplan-Meier survival estimates by MPR quartile are shown in Figure 1. Cumulative survival rates were $0.89(95 \% \mathrm{Cl}: 0.80-0.99)$ for the first quartile, $0.87(95 \% \mathrm{Cl}: 0.78-0.97)$ for the second, $0.84(95 \% \mathrm{Cl}: 0.73-0.95)$ for the third, and 0.76 (95\% Cl: 0.63-0.89) for the fourth. Overall, the survival estimates tended to decrease with increasing MPR, which was confirmed by the log-rank test $(p<0.001)$. 
Table I. Baseline characteristics of community-acquired pneumonia patients

\begin{tabular}{|c|c|c|c|c|}
\hline Baseline variables & Patients & Survival & Non-survival & $P$-value \\
\hline$n(\%)$ & $3665(100)$ & 3074 (83.9) & $591(16.1)$ & - \\
\hline Age, median (IQR) [years] & $74.0(65.0-80.0)$ & $74.0(64.0-80.0)$ & $75.0(69.0-81.0)$ & - \\
\hline Male, $n(\%)$ & $2351(64.1)$ & $1960(63.8)$ & $391(66.2)$ & 0.281 \\
\hline Female, $n(\%)$ & $1,314(35.9)$ & $1114(36.2)$ & $200(33.8)$ & \\
\hline \multicolumn{5}{|l|}{ Underlying disease, $n(\%)$ : } \\
\hline Hypertension & $1036(28.3)$ & $911(29.6)$ & $125(21.2)$ & $<0.001^{\star}$ \\
\hline Diabetes mellitus & $288(7.9)$ & $253(8.2)$ & $35(5.9)$ & 0.055 \\
\hline Cerebrovascular accident & $163(4.4)$ & $145(4.7)$ & $18(3.0)$ & 0.081 \\
\hline Cardiovascular disease & $121(3.3)$ & $94(3.1)$ & $27(4.6)$ & 0.077 \\
\hline Hepatic disease & $130(5.5)$ & $111(3.6)$ & $19(3.2)$ & 0.710 \\
\hline Renal disease & $248(6.8)$ & $203(6.6)$ & $45(7.6)$ & 0.371 \\
\hline Malignancy & $246(6.7)$ & $191(6.2)$ & $55(9.3)$ & $0.009^{*}$ \\
\hline \multicolumn{5}{|l|}{ Other respiratory disease, $n(\%)$ : } \\
\hline Asthma & $142(3.9)$ & $132(4.3)$ & $10(1.7)$ & $0.002^{*}$ \\
\hline Bronchitis & $167(4.6)$ & $148(4.8)$ & $19(3.2)$ & 0.105 \\
\hline COPD & $416(11.4)$ & $365(11.9)$ & $51(8.6)$ & $0.023^{*}$ \\
\hline Tuberculosis & $119(3.2)$ & $101(3.3)$ & $18(3.0)$ & 0.899 \\
\hline \multicolumn{5}{|l|}{ Clinical findings, mean \pm SD: } \\
\hline Systolic blood pressure [mm Hg] & $121 \pm 26.5$ & $122.2 \pm 25.5$ & $114.6 \pm 30.8$ & $<0.001^{*}$ \\
\hline Diastolic blood pressure [mm Hg] & $70.2 \pm 15.6$ & $71.0 \pm 15.2$ & $65.9 \pm 17.0$ & $0.025^{*}$ \\
\hline Respiration rate [min] & $20.9 \pm 4.0$ & $20.7 \pm 3.7$ & $22.0 \pm 5.0$ & $<0.001^{*}$ \\
\hline Pulse rate [min] & $96.1 \pm 21.1$ & $94.7 \pm 20.2$ & $103.7 \pm 24.4$ & $<0.001^{*}$ \\
\hline Body temperature $\left[{ }^{\circ} \mathrm{C}\right]$ & $37.1 \pm 0.8$ & $37.2 \pm 0.8$ & $37.0 \pm 0.9$ & 0.611 \\
\hline \multicolumn{5}{|l|}{ Mental status, $n(\%):$} \\
\hline Alert & $1081(29.4)$ & $979(31.8)$ & $102(17.3)$ & \multirow[t]{2}{*}{$0.024^{*}$} \\
\hline Altered & $2584(70.6)$ & $2095(68.2)$ & $489(83.7)$ & \\
\hline \multicolumn{5}{|l|}{ Bacteraemia, $n(\%)$ : } \\
\hline Yes & $2491(68.0)$ & $2032(66.1)$ & $459(77.7)$ & \multirow[t]{2}{*}{$<0.001^{*}$} \\
\hline No & $1174(32.0)$ & $1042(33.9)$ & $132(22.3)$ & \\
\hline \multicolumn{5}{|l|}{ ICU stay, $n(\%):$} \\
\hline Yes & $1379(37.6)$ & $963(31.3)$ & $416(70.4)$ & \multirow[t]{2}{*}{$<0.001^{*}$} \\
\hline No & $2286(62.4)$ & $2111(68.7)$ & $175(29.6)$ & \\
\hline \multicolumn{5}{|l|}{ Laboratory findings: } \\
\hline White blood cell, median (IQR) [× 109/I] & $10.9(7.3-15.3)$ & $10.9(7.6-15.1)$ & $11.1(6.0-16.3)$ & 0.177 \\
\hline Haemoglobin, mean \pm SD $[g / d l]$ & $11.6 \pm 2.2$ & $11.7 \pm 2.2$ & $11.3 \pm 2.3$ & 0.088 \\
\hline Platelet, median (IQR) [× 109/I] & $\begin{array}{c}222.0 \\
(159.0-308.0)\end{array}$ & $\begin{array}{c}228.0 \\
(167.0-312.8)\end{array}$ & $\begin{array}{c}191.5 \\
(112.0-265.3)\end{array}$ & $<0.001^{*}$ \\
\hline MPV, median (IQR) [fI] & $7.9(7.3-8.6)$ & $7.8(7.2-8.5)$ & $8.2(7.5-9.1)$ & $<0.001^{*}$ \\
\hline DNI, median (IQR) (\%) & $1.4(0.0-3.7)$ & $1.3(0.0-3.3)$ & $2.3(0.2-7.0)$ & $<0.001^{*}$ \\
\hline CRP, median (IQR) $[\mathrm{mg} / \mathrm{dl}]$ & $76.0(10.8-145.0)$ & $74.2(10.8-142.0)$ & $85.2(10.6-156.9)$ & 0.102 \\
\hline ESR, median (IQR) [mm/h] & $45.5(23.0-68.0)$ & $46.0(25.0-68.0)$ & $40.0(12.0-64.0)$ & $0.001^{\star}$ \\
\hline Procalcitonin, median (IQR) [ng/ml] & $0.6(0.2-3.5)$ & $0.5(0.2-2.7)$ & $1.7(0.4-9.3)$ & $<0.001^{*}$ \\
\hline
\end{tabular}

${ }^{*} P$-value $<0.05$ represents statistical significance. CAP - community-acquired pneumonia, IQR - interquartile range, COPD - chronic obstructive pulmonary disease, SD - standard deviation, ICU - intensive care unit, MPV - mean platelet volume, DNI - delta neutrophil index, CRP - C-reactive protein, ESR - erythrocyte sedimentation rate. 


\section{Comparisons between mean platelet volume to platelet count ratio values and CURB-65 and pneumonia severity index scores}

Additionally, among the 3665 study patients, we further made a comparison between MPR and other potential predictors of CAP, such as CURB-65 and PSI score. As mentioned above, only 150 patients had sufficient data to allow us to calculate CURB-65 and PSI scores, so we compared the MPR and these predictors (Table $\mathrm{V}$ ).

The fourth quartile of MPR was found to be associated with higher 60-day mortality than an MPR quartile of $\leq 3$ (37.5\% vs. $14.9 \%, p=0.002$ ). A PSI class of $\geq 4$ and a CURB- 65 score of $\geq 3$ were both associated with a greater risk of mortality (42.0\%, $p<0.001$ and $30.5 \%, p=0.006$, respectively). In terms of predicting 60-day mortality, the fourth quartile of MPR showed a sensitivity of $60.0 \%$ and a specificity of $69.6 \%$, CURB- 65 a sensitivity of $60 \%$ and a specificity of $74.8 \%$, and PSI a sensitivity of $82.9 \%$ and a specificity of $42.6 \%$.

In addition, ICU admission rates and bacteraemia rates were evaluated. For ICU admission rates, MPR showed a sensitivity of $55.4 \%$ and a specificity of $76.3 \%$, CURB- 65 a sensitivity of $51.4 \%$ and a specificity of $84.2 \%$, and PSI a sensitivity of $77 \%$ and a specificity of $50 \%$. For bacteraemia, MPR showed a sensitivity of $35.2 \%$ and a specificity of $57.1 \%$, CURB- 65 a sensitivity of $37 \%$ and a specificity of $76.2 \%$, and PSI class a sensitivity of $64.8 \%$ and a specificity of $40.5 \%$.

\section{Discussion}

The results of this study suggest that MPR is positively correlated with short-term mortality in CAP patients. This finding is consistent with those of previous studies, in which it was suggested that thrombocytopaenia and/or an elevated MPV had predictive value in patients with sepsis or CAP [22, 23, 25, 29].

In this study, the existence of medical histories seems to be an independent factor with regard to 60-day mortality. This is contrary to the results from other previous studies [30-32]. However, malignancy was more frequent, and altered mental status rate, bacteraemia rate, and ICU admission rate were higher for non-survivors. To minimise statistical errors caused by medical histories or laboratory results, we carried out logistic regression analyses after adjusting for these confounders.

Platelets play an important role in haemostasis, and their production is regulated by thrombopoietin via a feedback mechanism [33]. The primary role of platelets is thought to be related to the repair of injured blood vessels, aggregation and blood clot formation, and chemotaxis [34].
Table II. Receiver operating characteristics curve analysis

\begin{tabular}{|lcc|}
\hline Parameter & \multicolumn{2}{c|}{ Mortality } \\
\cline { 2 - 3 } & AUC & $95 \% \mathrm{Cl}$ \\
\hline WBC & 0.54 & $0.51-0.57$ \\
\hline DNI & 0.61 & $0.59-0.64$ \\
\hline CRP & 0.52 & $0.49-0.55$ \\
\hline ESR & 0.54 & $0.52-0.57$ \\
\hline Procalcitonin & 0.65 & $0.63-0.67$ \\
\hline NLR & 0.53 & $0.50-0.56$ \\
\hline MPR & 0.63 & $0.60-0.65$ \\
\hline
\end{tabular}

$W B C$ - white blood cell, DNI - delta neutrophil index, CRP - C-reactive protein, ESR - erythrocyte sedimentation rate, NLR - neutrophil to lymphocyte ratio, MPR - mean platelet volume to platelet count ratio.

Furthermore, several studies have shown activated platelets release cytokines and immunomodulators and are thus associated with innate and adaptive immunity [34-36]. One study showed that some platelet surface receptors react with exogenic bacteria and activate internal signalling resulting in platelet activation, complement fixation, and platelet aggregation [37], and another reported that activated platelets bind to circulating leukocytes in patients with sepsis and multiple organ failure [38].

The prognostic values of MPV and PC have been assessed in many diseases. MPV is a routinely measured parameter that provides a measure of average platelet size in blood samples. High MPV values have been reported to be associated with platelet activation and inflammatory processes [24], and for this reason MPV has been investigated in thromboembolic diseases, ischaemic cardiovascular diseases, steatohepatic diseases, and even in malignancies [39-41]. On the other hand, PC has been studied in CAP, sepsis, septic shock, and thrombocytopaenia, and several studies have shown that thrombocytopaenia is an independent hallmark in ICU patients with the prognosis of sepsis [23, 42, 43].

Moreover, MPR has been investigated in many studies. For example, several studies have investigated relations between MPR and disease severity and/or mortality in hepatocellular carcinoma [40], non-small cell lung cancer [39], and non-STelevation myocardial infarction [41]. And one study found that MPR was correlated with 30-day mortality in patients with pneumonia following ischaemic stroke [44]. Thus, associations have been reported between MPR and disease prognosis and/or mortality, but not between MPR and prognosis and mortality in CAP. 
Table III. Patient characteristics according to quartiles of mean platelet volume to platelet count ratio

\begin{tabular}{|c|c|c|c|c|c|}
\hline Baseline variables & Q1 & Q2 & Q3 & Q4 & $P$-value \\
\hline$n(\%)$ & $916(25.0)$ & $916(25.0)$ & $916(25.0)$ & $917(25.0)$ & - \\
\hline Age, median (IQR) [years] & $74.0(66.3-79.0)$ & $75.0(66.0-81.0)$ & $74.0(65.3-81.0)$ & $73.0(61.0-81.0)$ & - \\
\hline Male, $n(\%)$ & $566(61.8)$ & $577(63.0)$ & $595(65.0)$ & $613(66.8)$ & 0.116 \\
\hline Female, $n(\%)$ & $350(38.2)$ & $339(37.0)$ & $321(35.0)$ & $304(33.2)$ & \\
\hline \multicolumn{6}{|l|}{ Underlying disease, $n$ (\%): } \\
\hline Hypertension & $273(29.8)$ & $292(31.9)$ & $255(27.8)$ & $216(23.6)$ & 0.757 \\
\hline Diabetes mellitus & $69(7.5)$ & $78(8.5)$ & $71(7.8)$ & $70(7.6)$ & 0.863 \\
\hline Cerebrovascular accident & $33(3.6)$ & $55(6.0)$ & $42(4.6)$ & $33(3.6)$ & 0.453 \\
\hline Cardiovascular disease & $21(2.3)$ & $32(3.5)$ & $46(5.0)$ & $22(2.4)$ & 0.474 \\
\hline Hepatic disease & $28(3.1)$ & $23(2.5)$ & $22(2.4)$ & $57(6.2)$ & 0.872 \\
\hline Renal disease & $35(3.8)$ & $53(5.8)$ & $70(7.6)$ & $90(9.8)$ & $<0.001^{*}$ \\
\hline Malignancy & $81(8.8)$ & $52(5.7)$ & $60(6.6)$ & $53(5.8)$ & 0.203 \\
\hline \multicolumn{6}{|l|}{ Other respiratory disease, $n(\%)$ : } \\
\hline Asthma & $40(4.4)$ & $48(5.2)$ & $34(3.7)$ & $20(2.2)$ & 0.325 \\
\hline Bronchitis & $63(6.9)$ & $49(5.3)$ & $34(3.7)$ & $21(2.3)$ & 0.332 \\
\hline COPD & $118(12.9)$ & $129(14.1)$ & $110(12.0)$ & $59(6.4)$ & 0.496 \\
\hline Tuberculosis & $42(4.6)$ & $32(3.5)$ & $28(3.1)$ & $17(1.9)$ & 0.466 \\
\hline \multicolumn{6}{|l|}{ Clinical findings, mean \pm SD: } \\
\hline Systolic blood pressure [mm Hg] & $122.9 \pm 26.4$ & $123.0 \pm 25.5$ & $120.8 \pm 25.8$ & $117.4 \pm 28.1$ & 0.490 \\
\hline Diastolic blood pressure [mm Hg] & $71.2 \pm 14.8$ & $71.5 \pm 14.6$ & $70.3 \pm 16.3$ & $67.7 \pm 16.2$ & 0.536 \\
\hline Respiration rate [/min] & $20.9 \pm 4.1$ & $21.0 \pm 3.9$ & $20.7 \pm 3.7$ & $20.9 \pm 4.1$ & 0.438 \\
\hline Pulse rate $[/ \mathrm{min}]$ & $98.1 \pm 20.9$ & $94.8 \pm 20.2$ & $94.1 \pm 21.3$ & $97.1 \pm 21.9$ & 0.862 \\
\hline Body temperature $\left[{ }^{\circ} \mathrm{C}\right]$ & $37.1 \pm 0.8$ & $37.1 \pm 0.8$ & $37.2 \pm 0.8$ & $37.1 \pm 0.9$ & 0.848 \\
\hline \multicolumn{6}{|l|}{ Mental status, $n(\%)$ : } \\
\hline Alert & $200(21.8)$ & $267(29.1)$ & $285(31.1)$ & $329(35.9)$ & \multirow[t]{2}{*}{0.217} \\
\hline Altered & $716(78.2)$ & $649(70.9)$ & $631(68.9)$ & $588(64.1)$ & \\
\hline \multicolumn{6}{|l|}{ Bacteraemia, $n(\%)$ : } \\
\hline Yes & $645(70.4)$ & $591(64.5)$ & $607(66.3)$ & $648(70.7)$ & \multirow[t]{2}{*}{0.716} \\
\hline No & $271(29.6)$ & $325(35.5)$ & $309(33.7)$ & $269(29.3)$ & \\
\hline \multicolumn{6}{|l|}{ ICU stay, $n(\%)$ : } \\
\hline Yes & $300(32.8)$ & $286(31.2)$ & $326(35.6)$ & $467(50.9)$ & \multirow[t]{2}{*}{$<0.001^{\star}$} \\
\hline No & $616(67.2)$ & $630(68.8)$ & $590(64.4)$ & $450(49.1)$ & \\
\hline \multicolumn{6}{|l|}{ Laboratory findings: } \\
\hline $\begin{array}{l}\text { White blood cell, } \\
\text { median }(\mathrm{IQR})\left[\times 10^{9} / \mathrm{I}\right]\end{array}$ & $13.4(9.5-17.9)$ & $11.7(8.6-16.2)$ & $10.0(7.0-13.8)$ & $7.9(4.9-12.0)$ & $<0.001^{*}$ \\
\hline Haemoglobin, mean \pm SD $[\mathrm{g} / \mathrm{dl}]$ & $11.5 \pm 2.0$ & $12.0 \pm 2.1$ & $11.9 \pm 2.1$ & $11.2 \pm 2.4$ & 0.994 \\
\hline Platelet, median $(\mathrm{IQR})\left[\times 10^{9} / \mathrm{I}\right]$ & $\begin{array}{c}383.0 \\
(331.0-453.5)\end{array}$ & $\begin{array}{c}258.0 \\
(235.0-281.0)\end{array}$ & $\begin{array}{c}190.0 \\
(173.0-208.0)\end{array}$ & $\begin{array}{c}116.0 \\
(78.0-140.8)\end{array}$ & $<0.001^{*}$ \\
\hline MPV, median (IQR) [fI] & $7.3(6.8-7.8)$ & $7.6(7.2-8.1)$ & $8.0(7.5-8.5)$ & $8.9(8.2-9.8)$ & $0.044^{*}$ \\
\hline DNI, median (IQR) (\%) & $1.3(0.0-2.9)$ & $1.3(0.0-3.1)$ & $1.4(0.0-3.9)$ & $2.0(0.0-5.8)$ & 0.420 \\
\hline CRP, median (IQR) $[\mathrm{mg} / \mathrm{dl}]$ & $76.5(11.1-143.0)$ & $77.8(11.5-140.0)$ & $74.0(10.8-136.5)$ & $74.8(9.1-157.0)$ & 0.993 \\
\hline ESR, median (IQR) [mm/h] & $61.0(42.0-81.8)$ & $49.0(27.5-70.0)$ & $39.0(21.0-59.0)$ & $31.0(12.0-54.0)$ & $<0.001^{*}$ \\
\hline Procalcitonin, median (IQR) [ng/ml] & $0.3(0.1-1.4)$ & $0.5(0.2-2.1)$ & $0.7(0.2-4.2)$ & $1.7(0.4-8.8)$ & 0.610 \\
\hline
\end{tabular}


Table IV. Univariate and multivariate logistic regression of association between mean platelet volume to platelet count ratio and 60-day mortality

\begin{tabular}{|lcccccc|}
\hline \multirow{2}{*}{ MPR quintiles } & \multicolumn{2}{c}{ Unadjusted OR } & \multicolumn{2}{c}{ Adjusted OR ${ }^{1 \dagger}$} & \multicolumn{2}{c|}{ Adjusted OR ${ }^{2 \varsigma}$} \\
\cline { 2 - 7 } & OR $(95 \% \mathrm{Cl})$ & $P$-value & OR $(95 \% \mathrm{Cl})$ & $P$-value & OR $(95 \% \mathrm{Cl})$ & $P$-value \\
\hline First quartile & 1 & - & 1 & - & 1 & - \\
\hline Second quartile & $1.16(0.87-1.55)$ & 0.307 & $1.20(0.90-1.60)$ & 0.224 & $1.16(0.87-1.55)$ & 0.320 \\
\hline Third quartile & $1.48(1.12-1.95)$ & $0.006^{*}$ & $1.49(1.13-1.97)$ & $0.005^{*}$ & $1.37(1.03-1.81)$ & $0.030^{*}$ \\
\hline Fourth quartile & $2.93(2.27-3.78)$ & $<0.001^{*}$ & $3.06(2.36-3.96)$ & $<0.001^{*}$ & $2.66(2.04-3.46)$ & $<0.001^{\star}$ \\
\hline
\end{tabular}

${ }^{\star} P$-value $<0.05$ represents statistical significance. ${ }^{\dagger}$ Adjusted for age, history of hypertension, diabetes mellitus, cerebrovascular accident, cardiovascular disease, hepatic disease, renal disease, and malignancy. ${ }^{\S}$ Adjusted for age, sex, history of hypertension, diabetes mellitus, atrial fibrillation, heart failure, coronary artery occlusive disease, and malignancy plus white blood cell, erythrocyte sedimentation rate, delta neutrophil index, and procalcitonin. MPR - mean platelet volume to platelet count ratio, OR-odds ratio, $\mathrm{Cl}$ - confidence interval.

As mentioned above, it is known that a high MPV and a low PC are associated with prognosis in patients with CAP, sepsis, or septic shock, and thus we hypothesised that MPR might associated with disease prognosis in CAP. When the prognostic values of MPR quartiles were examined, we found that the fourth quartile significantly predicted short-term (60-day) mortality in CAP patients. In addition, univariate and multivariate logistic regression analysis revealed that the fourth and fifth MPR quartiles exhibited statistical significance as compared with the first quartile $(p<0.05)$. Kaplan-Meier survival analysis also showed that survival estimates significantly reduced with increasing MPR, and this finding was confirmed by log-rank testing $(p<0.05)$. Furthermore, these results are consistent with the findings of others for inflammatory diseases [39-41].

In this study, we suggest that MPR can be used as a predictor of mortality in CAP patients rather than either MPV or PC alone. MPR has a higher AUC value than that of MPV or PC (data not

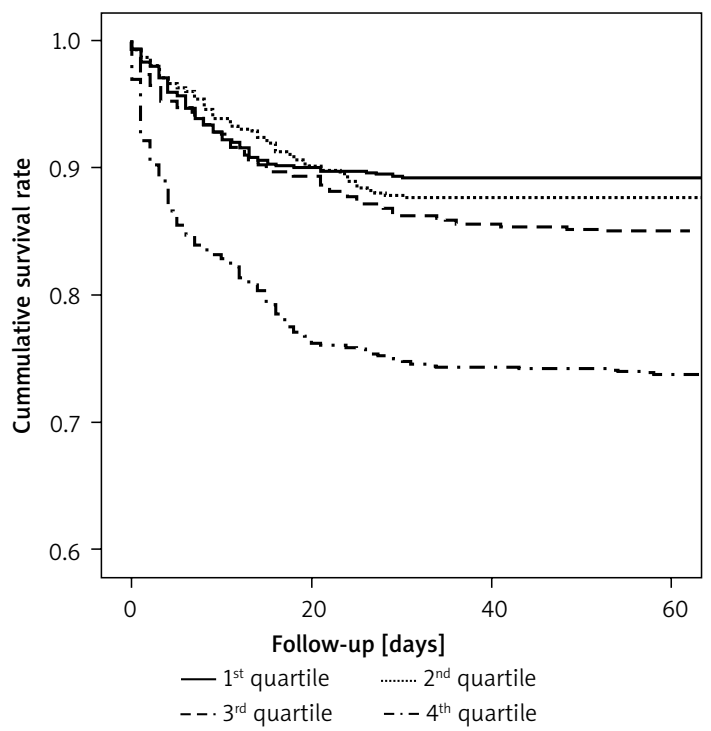

Figure 1. Kaplan-Meier survival estimates of community-acquired pneumonia patients according to quintiles of mean platelet volume to platelet count ratio

Table V. Comparison of prognostic performance among CURB-65, PSI class, and mean platelet volume to platelet count ratio

\begin{tabular}{|lccccc|}
\hline Parameter & Score & Patients & Survival & Non-survival & $P$-value \\
\hline MPR & $1-3$ & 94 & $80(85.1)$ & $14(14.9)$ & $0.002^{*}$ \\
\cline { 2 - 5 } & 4 & 56 & $35(62.5)$ & $21(37.5)$ & \\
\hline CURB-65 & $0-2$ & 100 & $86(86.0)$ & $14(14.0)$ & $<0.001^{*}$ \\
\cline { 2 - 5 } & $3-4$ & 50 & $29(58.0)$ & $21(42.0)$ & \\
\hline PSI class & $1-3$ & 55 & $49(89.1)$ & $6(10.1)$ & $0.006^{*}$ \\
\cline { 2 - 5 } & $4-5$ & 95 & $66(69.5)$ & $29(30.5)$ & \\
\hline
\end{tabular}

\begin{tabular}{|c|c|c|c|c|c|c|}
\hline \multirow[t]{2}{*}{ Parameter } & \multicolumn{2}{|c|}{ 60-day mortality } & \multicolumn{2}{|c|}{ Intensive care unit stay } & \multicolumn{2}{|c|}{ Bacteremia } \\
\hline & Sensitivity & Specificity & Sensitivity & Specificity & Sensitivity & Specificity \\
\hline MPR & $60.0(43.6-74.4)$ & $69.6(60.6-77.2)$ & $55.4(44.1-66.2)$ & $76.3(65.6-84.5)$ & $35.2(26.8-44.6)$ & $57.1(42.2-70.9)$ \\
\hline CURB-65 & $60.0(43.6-74.4)$ & $74.8(66.1-81.8)$ & $51.4(40.2-62.4)$ & $84.2(74.4-90.7)$ & $37.0(28.5-46.4)$ & $76.2(61.5-86.5)$ \\
\hline PSI class & $82.9(67.3-91.9)$ & $42.6(34.0-51.7)$ & $77.0(66.3-85.1)$ & $50.0(39.0-61.0)$ & $64.8(55.4-73.2)$ & $40.5(27.0-55.5)$ \\
\hline
\end{tabular}

CURB-65 - confusion, urea, respiration, blood pressure, age > 65 years; PSI - pneumonia severity index, MPR - mean platelet volume to platelet count ratio. 
shown). Also in this study, we found that high MPV and low PC value were associated with 60 -day mortality. PC values can be affected by other confounding factors or diseases, so more reliable results can be obtained by using MPV values. Mean platelet volume to platelet count ratio has a relatively high AUC value, which was similar to the AUC value of procalcitonin. Without the need to measure procalcitonin or other markers, we can simply predict disease-related mortality in CAP patients with only MPV and PC values that are measured on a routine $C B C$ analysis.

Additionally, the prognostic values of MPR and other pneumonia severity scales (CURB-65 and PSI) for 60-day mortality, ICU admission, and bacteraemia MPR were comparable, and although the sensitivities of MPR and CURB-65 were lower than for PSI, their specificities were much higher. In fact, MPR and CURB-65 showed high specificity for predicting short-term mortality and ICU admission, but predictions of bacteraemia lacked significance.

Several limitations of the present study warrant a mention. First, the study is limited by its retrospective, non-randomised, single-centre design, and thus, further multi-centre studies are required to confirm our findings. Second, the confounding effects of factors such as concurrent cerebrovascular, cardiovascular, hepatic, and renal diseases, and malignancy were not fully assessed. Third, we did not include CAP-causing pathogen types in the analysis.

In conclusion, we found that elevated initial MPR correlated with 60-day mortality in patients with CAP. We conclude that MPR may provide a significant laboratory biomarker for predicting CAP-related mortality, and we suggest further prospective studies need to be conducted to further investigate and validate the use and exact role of MPR in CAP and other diseases.

\section{Conflict of interest}

The authors declare no conflict of interest.

\section{References}

1. Liu D, Su Lx, Guan W, Xiao K, Xie L. Prognostic value of procalcitonin in pneumonia: a systematic review and meta-analysis. Respirology 2016; 21: 280-8.

2. Huang DT, Weissfeld LA, Kellum JA, et al. Risk prediction with procalcitonin and clinical rules in community-acquired pneumonia. Ann Emerg Med 2008; 52: 48-58.e2.

3. Müller B, Harbarth S, Stolz D, et al. Diagnostic and prognostic accuracy of clinical and laboratory parameters in community-acquired pneumonia. BMC Infect Dis 2007; 7: 10 .

4. Choi MJ, Song JY, Noh JY, et al. Disease burden of hospitalized community-acquired pneumonia in South Korea: analysis based on age and underlying medical conditions. Medicine (Baltimore) 2017; 96: e8429.
5. Menéndez R, Martinez R, Reyes S, et al. Biomarkers improve mortality prediction by prognostic scales in community-acquired pneumonia. Thorax 2009; 64: 587-91.

6. Huang H, Ideh RC, Gitau E, et al. Discovery and validation of biomarkers to guide clinical management of pneumonia in African children. Clin Infect Dis 2014; 58: 1707-15.

7. Viasus D, Del Rio-Pertuz G, Simonetti AF, et al. Biomarkers for predicting short-term mortality in communityacquired pneumonia: a systematic review and metaanalysis. J Infect 2016; 72: 273-82.

8. Yoon NB, Son C, Um SJ. Role of the neutrophil-lymphocyte count ratio in the differential diagnosis between pulmonary tuberculosis and bacterial communityacquired pneumonia. Ann Lab Med 2013; 33: 105-10.

9. Kim MW, Lim JY, Oh SH. Mortality prediction using serum biomarkers and various clinical risk scales in community-acquired pneumonia. Scand J Clin Lab Invest 2017; 77: 486-92.

10. Park JH, Wee JH, Choi SP, Oh SH. The value of procalcitonin level in community-acquired pneumonia in the ED. Am J Emerg Med 2012; 30: 1248-54.

11. Berg P, Lindhardt $B \varnothing$. The role of procalcitonin in adult patients with community-acquired pneumonia - a systematic review. Dan Med J 2012; 59: A4357.

12. Ito A, Ishida T, Tachibana H, Ito Y, Takaiwa T. Serial procalcitonin levels for predicting prognosis in community-acquired pneumonia. Respirology 2016; 21: 1459-64.

13. Hirakata Y, Yanagihara K, Kurihara S, et al. Comparison of usefulness of plasma procalcitonin and C-reactive protein measurements for estimation of severity in adults with community-acquired pneumonia. Diagn Microbiol Infect Dis 2008; 61: 170-4.

14. Krüger S, Welte T. Biomarkers in community-acquired pneumonia. Expert Rev Respir Med 2012; 6: 203-14.

15. Nseir WB, Mograbi JM, Amara AE, Abu Elheja OH, Mahamid MN. Non-alcoholic fatty liver disease and 30-day all-cause mortality in adult patients with community-acquired pneumonia. QJM 2019; 112: 95-9.

16. Schuetz P, Widmer I, Chaudri A, Christ-Crain M, Zimmerli W, Mueller B. Prognostic value of procalcitonin in community-acquired pneumonia. Eur Respir J 2011; 37: 384-92.

17. Masiá M, Gutiérrez F, Shum C, et al. Usefulness of procalcitonin levels in community-acquired pneumonia according to the patients outcome research team pneumonia severity index. Chest 2005; 128: 2223-9.

18. Williams DJ, Hall M, Auger KA, et al. Association of white blood cell count and c-reactive protein with outcomes in children hospitalized for community-acquired pneumonia. Pediatr Infect Dis J 2015; 34: 792-3.

19. Zhydkov A, Christ-Crain M, Thomann R, et al. Utility of procalcitonin, C-reactive protein and white blood cells alone and in combination for the prediction of clinical outcomes in community-acquired pneumonia. Clin Chem Lab Med 2015; 53: 559-66.

20. de Jager CP, van Wijk PT, Mathoera RB, de Jongh-Leuvenink J, van der Poll T, Wever PC. Lymphocytopenia and neutrophil-lymphocyte count ratio predict bacteremia better than conventional infection markers in an emergency care unit. Crit Care 2010; 14: R192.

21. Lee JH, Song S, Yoon SY, Lim CS, Song JW, Kim HS. Neutrophil to lymphocyte ratio and platelet to lymphocyte ratio as diagnostic markers for pneumonia severity. Br J Biomed Sci 2016; 73: 140-2.

22. Mirsaeidi M, Peyrani P, Aliberti S, et al. Thrombocytopenia and thrombocytosis at time of hospitalization 
predict mortality in patients with community-acquired pneumonia. Chest 2010; 137: 416-20.

23. Brogly N, Devos P, Boussekey N, Georges H, Chiche A Leroy O. Impact of thrombocytopenia on outcome of patients admitted to ICU for severe community-acquired pneumonia. J Infect 2007; 55: 136-40.

24. Kim CH, Kim SJ, Lee MJ, et al. An increase in mean platelet volume from baseline is associated with mortality in patients with severe sepsis or septic shock. PLoS One 2015; 10: e0119437.

25. Karadag-Oncel E, Ozsurekci Y, Kara A, Karahan S, Cengiz AB, Ceyhan M. The value of mean platelet volume in the determination of community acquired pneumonia in children. Ital J Pediatr 2013; 39: 16.

26. Niederman MS, Mandell LA, Anzueto A, et al. Guidelines for the management of adults with communityacquired pneumonia: diagnosis, assessment of severity, antimicrobial therapy, and prevention. Am J Respir Crit Care Med 2001; 163: 1730-54.

27. Schuetz P, Suter-Widmer I, Chaudri A, Christ-Crain M, Zimmerli W, Mueller B. Prognostic value of procalcitonin in community-acquired pneumonia. Eur Respir J 2011; 37: 384-92.

28. Dellit TH, Owens RC, McGowan JE Jr, et al. Infectious Diseases Society of America and the Society for Healthcare Epidemiology of America guidelines for developing an institutional program to enhance antimicrobial stewardship. Clin Infect Dis 2007; 44: 159-77.

29. Aydemir H, Piskin N, Akduman D, Kokturk F, Aktas E. Platelet and mean platelet volume kinetics in adult patients with sepsis. Platelets 2015; 26: 331-5.

30. Budzynski J, Tojek K, Wustrau B, et al. The "cholesterol paradox" among inpatients - retrospective analysis of medical documentation. Arch Med Sci Atheroscler Dis 2018; 3: e46-57.

31. Arundel C, Sheriff H, Bearden DM, et al. Discharge home health services referral and 30-day all-cause readmission in older adults with heart failure. Arch Med Sci 2018; 14: 995-1002.

32. Soz G, Karakaya Z. The evaluation of geriatric patients who presented with trauma to the emergency department. Arch Med Sci 2019; 15: 1261-8.

33. de Graaf CA, Metcalf D. Thrombopoietin and hematopoietic stem cells. Cell Cycle 2011; 10: 1582-9.

34. Ferdous F, Scott TR. A comparative examination of thrombocyte/platelet immunity. Immunol Lett 2015; 163: 32-9.

35. Elzey BD, Sprague DL Ratliff TL The emerging role of platelets in adaptive immunity. Cell Immunol 2005; 238: 1-9.

36. Elzey BD, Tian J, Jensen RJ, et al. Platelet-mediated modulation of adaptive immunity. A communication link between innate and adaptive immune compartments. Immunity 2003; 19: 9-19.

37. Fitzgerald JR, Foster TJ, Cox D. The interaction of bacterial pathogens with platelets. Nat Rev Microbiol 2006; 4: 445-57.

38. Gawaz M, Fateh-Moghadam S, Pilz G, Gurland HJ, Werdan K. Platelet activation and interaction with leucocytes in patients with sepsis or multiple organ failure. Eur J Clin Invest 1995; 25: 843-51.

39. Inagaki N, Kibata K, Tamaki T, Shimizu T, Nomura S. Prognostic impact of the mean platelet volume/platelet count ratio in terms of survival in advanced non-small cell lung cancer. Lung Cancer 2014; 83: 97-101.

40. Cho SY, Yang JJ, You E, et al. Mean platelet volume/platelet count ratio in hepatocellular carcinoma. Platelets 2013; 24: 375-7.
41. Azab B, Torbey E, Singh J, et al. Mean platelet volume/ platelet count ratio as a predictor of long-term mortality after non-ST-elevation myocardial infarction. Platelets 2011; 22: 557-66.

42. Venkata C, Kashyap R, Farmer JC, Afessa B. Thrombocytopenia in adult patients with sepsis: incidence, risk factors, and its association with clinical outcome. J Intensive Care 2013; 1: 9.

43. Vanderschueren S, De Weerdt A, Malbrain M, et al. Thrombocytopenia and prognosis in intensive care. Crit Care Med 2000; 28: 1871-6.

44. Nageeb RS, Abozaid MMN, Nageeb GS, Omran AA. Mean platelet volume to platelet count ratio as a laboratory indicator of mortality in pneumonia following ischemic stroke. Egypt J Neurol Psychiatr Neurosurg 2018; 54: 27. 